\title{
CO2 laser ablation of oropharyngeal dysplasia
}

\author{
Waseem Jerjes* ${ }^{*}$ Tahwinder Upile, Zaid Hamdoon, Colin Hopper \\ From 2nd Scientific Meeting of the Head and Neck Optical Diagnostics Society \\ San Francisco, CA, USA. 23-24 January 2010
}

\section{Introduction}

The use of $\mathrm{CO}_{2}$ laser in the management of oral dysplastic lesions have been put into practice for more than a few years now. The main advantage is the decrease in local tissue morbidity. Very few studies have evaluated recurrence, malignant transformation and overall outcome in patients undergoing such procedure.

\section{Materials and methods}

In this prospective study, a total of 123 oral dysplastic lesions from 77 consecutive patients were treated with $\mathrm{CO}_{2}$ laser (resection and/or ablation). The average age was $58 \pm 4.8$ years. Patients' recovery was uneventful and no complications reported. Comparisons with the clinical and histopathological features and rate of recurrence as well as malignant transformation were made. These patients were followed-up for a mean of 6.4 years, and biopsies taken in case of changes indicative of malignant development.

\section{Analysis and results}

The results were analysed by an independent statistician using SPSS 17. The results were cross tabulated and the Chi-squared statistic was used to test for differences in the case-mix.

Homogenous leukoplakias were identified in 31 patients, non-homogenous leukoplakias in 34 patients, whereas 12 patients had erythroplakias. Ex- and lifelong smokers formed $88.3 \%$ of the recruited patients. While people who currently smoke and drink formed $55.8 \%$ (43 patients) of the cohort. Erythroplakias were solely identified in heavy lifelong smokers. The most common identified primary anatomical locations were the lateral border of tongue, floor of mouth and buccal mucosa. Moderate dysplasia was identified in 42 patients while 18 patients had severe dysplasias.
Laser resection margins in selected cases (68 patients) were clear in 53 and showed mild-moderate dysplasia in the involved margins. The rate of recurrence had no significant association with the location or the severity of epithelial dysplasia. The rate of first recurrence in laser surgery was approximately $19.5 \%$. Malignant transformation was observed in 8 patients $(10.4 \%)$, in the tongue and the floor of mouth. Recurrence and malignant transformation was mainly identified in erythroplakias and non-homogenous leukoplakias.

\section{Conclusion}

Recurrence and/or malignant transformation of oral dysplasia have been observed following laser surgery. Laser resection/ablation is recommended for oral dysplasia to prevent not only recurrence and malignant transformation, but also postoperative oral dysfunction encountered by other conventional modalities. In this study, smoking and drinking were associated with oral erythroplakia formation; the latter was identified to recur and transform into squamous cell carcinoma more than other lesions.

Published: 29 October 2010

doi:10.1186/1758-3284-2-S1-O34

Cite this article as: Jerjes et al:: CO2 laser ablation of oropharyngeal dysplasia. Head \& Neck Oncology 2010 2(Suppl 1):O34.
UCL Department of Surgery, University College London Medical School, London, UK 